\title{
Single shot XUV nanoimaging using an intense femtosecond soft X-ray laser
}

\author{
Michael Zürch ${ }^{1,2,3,{ }^{*}, \text { Frederik Tuitje }}{ }^{1}$, Tobias Helk $^{1}$, Julian Gautier ${ }^{4}$, Fabian Tissandier ${ }^{4}$, \\ Jean-Philippe Goddet ${ }^{4}$, Alexander Guggenmos ${ }^{3,5,6}$, Ulf Kleineberg ${ }^{5,6}$, Stephane Sebban ${ }^{4}$, \\ and Christian Spielmann ${ }^{1,2}$ \\ ${ }^{1}$ Institute of Optics and Quantum Electronics, Abbe Center of Photonics, Jena University, 07743 Jena, \\ Germany \\ ${ }^{2}$ Helmholtz Institute Jena, 07743 Jena, Germany \\ ${ }^{3}$ Department of Chemistry, University of California, Berkeley, CA 94720, USA \\ ${ }^{4}$ LOA, ENSTA, CNRS, Ecole Polytechnique, Université Paris-Saclay, F-91762 Palaiseau cedex, \\ France \\ ${ }^{5}$ Ludwig-Maximilians-Universität München, Am Coulombwall 1, 85748, Garching, Germany \\ ${ }^{6}$ Max-Planck-Institut für Quantenoptik, Hans-Kopfermann-Str. 1, 85748, Garching, Germany \\ *michael.zuerch@uni-jena.de
}

\begin{abstract}
We report the direct wavefront characterization of an intense ultrafast high-harmonic-seeded soft X-ray laser $(\lambda=32.8 \mathrm{~nm})$ depending on the arrival time of the seed pulses by high-resolution ptychographic imaging and subsequently perform single-shot nanoscale imaging.
\end{abstract}

\section{Introduction}

Employing coherent light with wavelengths substantially shorter than visible light is enabling high-resolution imaging beyond the limitations of the optical domain by means of coherent diffraction imaging. Large-scale X-ray Free-electron lasers are regularly employed for single-shot imaging at few nanometer resolution, while compact laboratory sources based on high harmonic generation (HHG) are getting increasingly popular, regularly reaching an imaging resolution near the wavelength limit [1,2]. Although HHG sources offer in principle excellent opportunities for imaging dynamic processes, i.e. time-resolved imaging, due to the femtosecond duration of the pulses, the low photon flux per pulse requires averaging over a substantial number of pulses and integration times in the order of several seconds are common. Further, the relatively large bandwidth of the HHG emission requires additional optics to sufficiently monochromatize the emission to achieve a temporal coherence sufficient for coherent diffraction imaging. In turn, soft X-ray lasers (SXRL) are table-top sources that offer a photon flux per laser shot that is sufficiently high for single shot imaging near wavelength limited resolution. In a SXRL, a high-energy pump pulse highly ionizes a solid or a gas until it reaches a nickel-like configuration that allows 
for a three-level lasing scheme, which is driven into population inversion by a second pump pulse. Depending on the chosen target medium, a SXRL emits intense laser radiation in the soft X-ray range within a narrow bandwidth corresponding to an atomic transition in the highly ionized medium. The pulse duration is in the order of a picosecond and thus a SXRL is ideally suited for time-resolved high-resolution imaging. However, it has been recently shown that a SXRL operating in an amplified spontaneous emission (ASE) scheme has a transverse coherence that severely limits the applicability for coherent imaging applications [3]. Fortunately, seeding a SXRL with a highly coherent HHG pulse, where a high harmonic emission line is precisely tuned to the atomic transition of the SXRL, has been realized and even shown pulse durations of several hundred femtoseconds accessing the realm of ultrafast single shot imaging [4]. In this scheme, the SXRL becomes a laser plasma amplifier for the HHG radiation. In such a high-harmonic-seeded SXRL the arrival time of the pump pulse that generates the population inversion and the HHG seed pulse needs to be precisely timed for reaching the highest gain in the laser plasma amplifier with few hundred femtosecond pulses.

Here, we investigate the wavefront properties of a high-harmonic-seeded SXRL depending on the time delay between the SXRL pump and HHG seed pulses. Specifically, we investigate the coherent wavefront directly at the output of the amplifying plasma channel for different time delays between the pump and seed pulses. For resolving the wavefront in amplitude and phase at the exit of the plasma channel, we image a micron scale target using ptychography and backpropagate the measured complex-valued illumination field to the exit of the plasma channel. Following characterization and optimization of the source properties, we successfully perform single shot nanoscale imaging.

\section{Experiment and results}

The experiment (Fig. 1a) was conducted at the Laboratoire d'Optique Appliquée with the 'Salle Jaune' Ti:Sapphire laser system pumping a SXRL using krypton gas producing $\mathrm{Kr}^{8+}$ ions in a $5 \mathrm{~mm}$ long plasma waveguide emitting at $32.8 \mathrm{~nm}$. The high harmonic seed is derived from a gas cell filled with argon. Details about the SXRL producing pulses with energies of $2 \mu \mathrm{J}$ per shot in a relative bandwidth better than 1/10000 can be found in [4]. The SXRL beam is separated from the optical beams by means of aluminum filters and reimaged using state-of-the-art curved multilayer mirrors (demagnification 1:10) onto a binary transmission sample consisting of a pattern of regular holes with $0.6 \mu \mathrm{m}$ diameter and a spacing of $1.0 \mu \mathrm{m}$ (see inset Fig. 1b) mounted in a $x-y-z$ scanner. A CCD camera captures the coherently diffracted light downstream (numerical aperture about 0.25 ) and the sample was raster scanned over 30 scan points for ptychographic reconstruction by an implementation of the extended ptychographic iterative engine (ePIE) algorithm using position refinement. At each scan point single shot exposure of the sample by the HHG-seeded SXRL is performed. This allows reconstructing a high-resolution image of the sample and, at the same time, the complex-valued illumination field from the SXRL at the sample (Fig. 1b). We employ an aliasing free Fresnel propagator to propagate the field back to the exit of the laser plasma amplifier. This experiment is repeated for several time delays $\tau$, where positive $\tau$ means the HHG seed pulse arrives following the SXRL pump pulse. For each delay step a full ptychographic reconstruction and backpropagation is performed, and, thus, allows quantifying the wavefront of the seeded SXRL out of the plasma channel in amplitude and phase. For a delay of 1.2 ps the highest photon flux is observed in the experiment, suggesting that highest gain is achieved in the plasma amplifier. The beam out of the laser plasma amplifier shows excellent spatial profiles (Fig. 1c depicting two 
representative time delays). For increasing time delay, a decreasing mode field diameter is observed, while at the same time the wavefront curvature is reduced. Following the characterization and optimization of the HHG-seeded SXRL we performed single-shot fullfield coherent diffraction imaging. Fig. 1d shows a typical recorded diffraction pattern that is successfully reconstructed (Fig. 1e) with a resolution in the order of $\sim 100 \mathrm{~nm}$ at few hundred femtosecond exposure time.
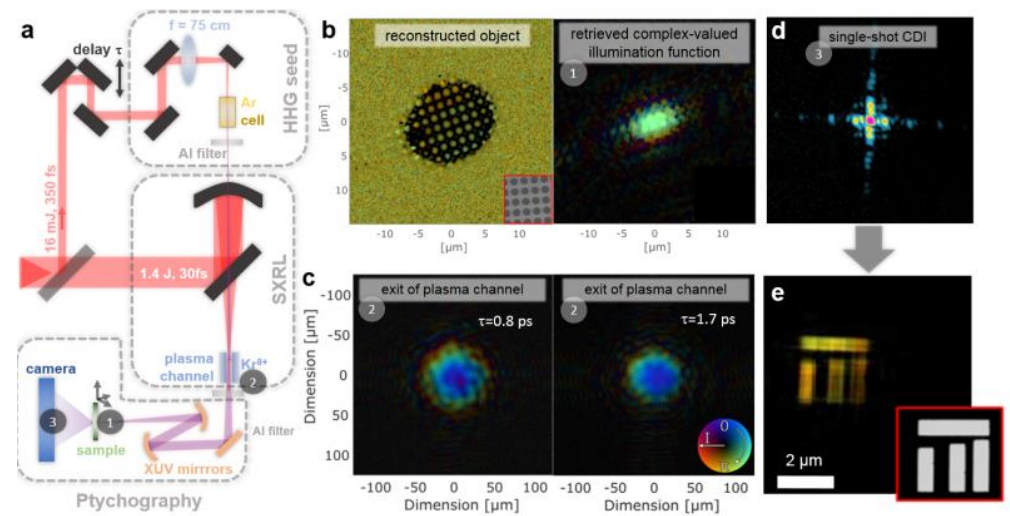

Fig. 1. Ptychographic characterization of a HHG-seeded SXRL. (a) Experimental scheme for ptychographic characterizing the beam of a HHG-seeded SXRL in amplitude and phase and singleshot ultrafast imaging (the plasma channel is created by another, for clarity not shown, arm of the same input laser). (b) Reconstruction of a microscopic object imaged by ptychography (inset shows SEM image of the sample used for ptychography) and reconstructed complex-valued illumination function at the sample. (c) Examples of measured normalized complex-valued SXRL beam profiles at the exit of the plasma channel for two different time delays between SXRL pump and HHG seed as indicated. (d) Measured single shot coherent diffraction pattern and (e) reconstruction of the object with $\sim 100 \mathrm{~nm}$ resolution (inset shows SEM image of the sample used for single shot measurements). All panels except (d) show complex-valued spatial representations where the amplitude and phase are encoded by brightness and hue as indicated in right panel in (c).

\section{Conclusions}

In conclusion, we successfully employed ptychographic coherent diffraction imaging for characterizing the beam of a HHG-seeded SXRL operating at $32.8 \mathrm{~nm}$ wavelength in amplitude and phase with high fidelity. Backpropagation of the field allows determining source properties in unprecedented quality. We find that HHG seeding results in excellent spatial coherence properties, while a high degree of temporal coherence is maintained through the narrow-band amplification. Further, we find that the time delay dependence between the pump and seed pulses causes significant reshaping of the amplified laser beam hinting at a complex seed-plasma interaction that is subject of current investigations. Finally, we successfully employ the characterized and optimized HHG-seeded SXRL for single-shot coherent diffraction imaging demonstrating the outstanding capabilities for ultrafast nanoscale imaging.

\section{References}

1. G.K. Tadesse, et al., Opt. Lett. 41 (22), 5170-5173 (2015).

2. M. Zürch, et al., Sci. Rep. 4, 7356 (2014).

3. M. Zürch, et al., Sci. Rep. 7, 5314 (2017).

4. A. Depresseux, et al., Nat. Photonics 9, 817 (2015). 